\title{
Array-scale inverse design of active metasurfaces
}

\author{
Prachi Thureja ${ }^{1}$, Ghazaleh Kafaie Shirmanesh ${ }^{1}$, Katherine T. Fountaine ${ }^{2}$, Ruzan Sokhoyan ${ }^{1}$, Meir \\ Grajower', Harry A. Atwater, ${ }^{1}$, \\ ${ }^{1}$ Thomas J. Watson Laboratories of Applied Physics, California Institute of Technology, Pasadena, California 91125, USA \\ ${ }^{2}$ Northrop Grumman NG Next, Redondo Beach, California 90278, USA \\ *haa@caltech.edu
}

\begin{abstract}
We develop an inverse design approach to optimize array architectures of reconfigurable metasurfaces and report dramatically improved beam steering performances with non-ideal antenna components. The versatility is enhanced by enabling continuous steering up to $70^{\circ}$. (C) 2020 The Author(s)
\end{abstract}

\section{Introduction}

In recent years, research on active metasurfaces has inspired the development of a new class of reconfigurable devices that enable the realization of dynamically tunable, functional arrays in a compact, lightweight chip format. Active control of the constitutive properties of light can be achieved by employing tunable metasurface elements that are actuated by external stimuli which modulate the complex permittivities of each element. The use of reconfigurable design elements has led to the implementation of 'universal' metasurfaces enabling multiple functions such as dynamic beam steering and reconfigurable light focusing by a single device [1]. However, the intrinsic tuning mechanism for each antenna element relies on resonances that lead to a non-ideal optical response consisting of a strong covariance of the scattered light phase and amplitude and a limited phase modulation. This fundamentally limits the performance of conventional 'forward' designed phased arrays that employ intuitively motivated phase profiles. For beam steering devices, the non-ideal response gives rise to significant power coupled into undesired sidelobes. Here, we develop high-performance active metasurfaces based on an array-scale 'inverse' design approach that overcomes these limitations, and has the potential to significantly increase the performance and versatility of state-of-the-art antenna arrays. In contrast to previously demonstrated inverse design approaches [2-4], our algorithm enhances array performances without the need of antenna-level device redesign which would require a complex fabrication procedure.
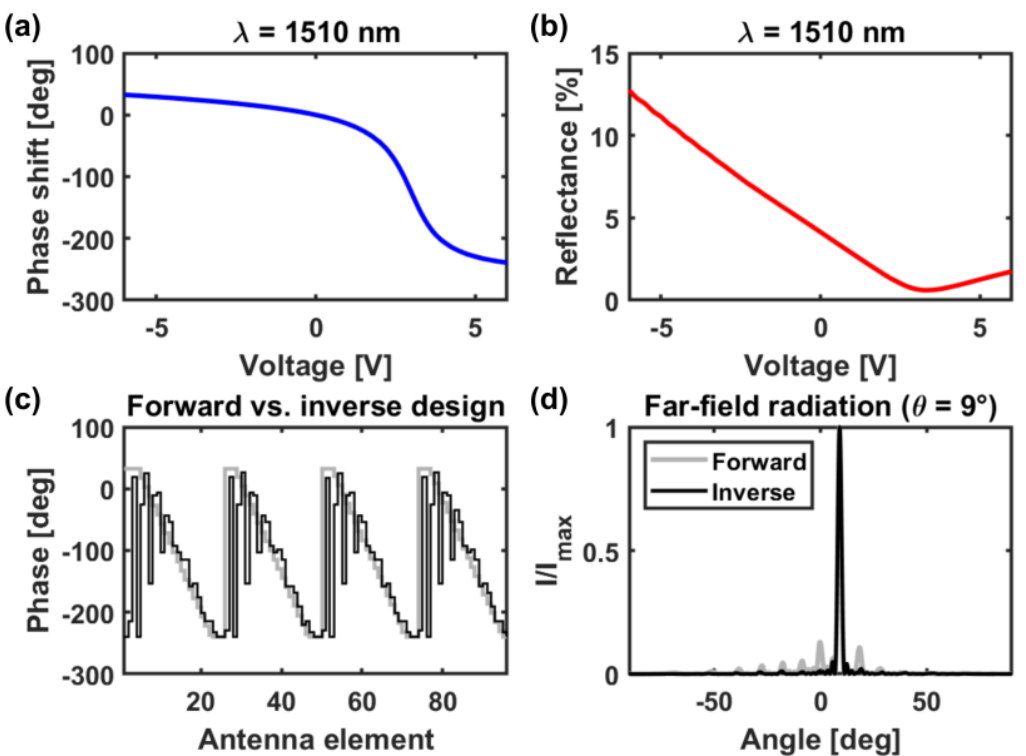

Fig. 1. (a) Phase and (b) amplitude of the scattered light characterized in terms of its reflectance for the previously demonstrated 'universal' metasurface. The optical response, simulated at a wavelength of $\lambda=1510 \mathrm{~nm}$, is tunable as a function of the applied bias voltage. [1] (c) Forward design (grey) using gradient phase profiles that are capped at minimal and maximal accessible phase values shown in (a) and inverse design (black) using the optimization algorithm developed in this work. (d) Far-field radiation pattern calculated using the optical response of the 'universal' metasurface as well as the forward- (grey) and inverse-designed phase profiles (black), respectively. 


\section{Requirements and challenges in the design of active metasurfaces}

For efficient beam steering and light focusing metasurfaces, each antenna element should ideally enable complete phase modulation over $360^{\circ}$ while the scattered wave amplitude remains constant. These ideal antenna characteristics would facilitate design of pure phase modulated metasurfaces which enable efficient wave front engineering with commonly used, 'forward' designs of the array properties. For beam steering, highly directive beams can be achieved by designing intuitive gradient phase profiles that cause the scattered light to constructively interfere in the desired direction. The underlying device physics of active metasurfaces, however, prevents the realization of pure phase gratings due to a non-ideal optical response that is characterized by a strongly covarying phase and amplitude, as shown in Fig. 1a-b for the electro-optically tunable, 'universal' metasurface introduced by Shirmanesh et al. [1]. In addition, actively tunable antenna arrays only exhibit limited phase modulations that demand adjustments in conventional, forward-designed phase profiles (Fig. 1c, grey line). These deviations from the ideal design therefore result in substantial undesired artefacts that manifest as sidelobes in the far-field radiation pattern of beam steering devices (Fig. 1d, grey line).

\section{Array-scale inverse design}

Here, we perform an array-scale inverse design of the actively tunable, 'universal' metasurface that exhibits a nonideal antenna response as described above. The designed optimization approach aims to find the optimal parameters (i.e. voltage/phase profiles) that efficaciously enable desired functionalities. The algorithm relies on an iterative genetic optimization to overcome the inherent high dimensionality of the problem that arises due to simultaneous optimization of 96 independently tunable antenna elements. By considering the variable interdependencies of the phase and amplitude values of the scattered light, the algorithm succeeds in generating non-intuitive array profiles (Fig. 1c, black line) that substantially enhance beam steering performances by suppressing sidelobes (Fig. 1d, black line). As a consequence, we are able to report a considerable increase in the directivity (a measure for the beam steering performance) of up to $84 \%$ compared to previously employed, forward designs in experimental demonstrations of the 'universal' metasurface. The optimized results that are obtained with strongly limited phase modulation $\left(\sim 270^{\circ}\right)$ correspond to far-field radiation patterns that visually and quantitatively approach ideal beam steering performances. Moreover, we substantially enhance the versatility of actively tunable antenna arrays by demonstrating continuous beam steering up to approximately $70^{\circ}$. The precise control over the amplitude and the direction of the steered beam further enables the implementation of a so-called beam splitting device that creates scattered beams that can simultaneously be steered into multiple directions.

The approach presented in this work paves the way to a multiscale inverse design of array, antenna and materials in reconfigurable metasurfaces that has the potential to enable versatile devices which efficiently achieve desired functionalities. The combined optimization will allow circumvention of the required stringency in the design of ideally performing individual components on a device-level and instead exploit the system-level flexibility to generate designs that optimally enable desired functionalities, as outlined in this work. This will ultimately remove the need for tedious fabrication procedures that otherwise would be required for complex metasurface antenna designs.

\section{Summary}

In conclusion, we introduced an array-scale inverse design approach that significantly enhances the performance and versatility of actively tunable metasurfaces consisting of non-ideal antenna components. The non-intuitive array designs are generated using highly non-intuitive phase profiles which consider the device-specific phase and amplitude relations. The strongly improved performances generated using this method support future studies towards a combined optimization of array, antenna and materials that will enable a new class of high-performing, multifunctional devices.

\section{References}

[1] G. K. Shirmanesh, R. Sokhoyan, P. C. Wu, and H. A. Atwater, "Electro-Optically Tunable Universal Metasurfaces," arXiv preprint arXiv:1910.02069 (2019).

[2] D. Sell, J. Yang, S. Doshay, R. Yang, and J. A. Fan, "Large-Angle, Multifunctional Metagratings Based on Freeform Multimode Geometries," Nano Lett. 17, 3752-3757 (2017).

[3] R. Pestourie, C. Pérez-Arancibia, Z. Lin, W. Shin, F. Capasso, and S. G. Johnson, "Inverse design of large-area metasurfaces," Opt. Express 26(26), $33732(2018)$.

[4] H. Chung and O. D. Miller, "Tunable metasurface inverse design for $80 \%$ switching efficiencies and $144^{\circ}$ angular steering," arXiv preprint arXiv:1910.03132 (2019). 